\title{
Video Rate Adaptation and Scheduling in Multi-rate Wireless Networks ${ }^{\star}$
}

\author{
Sourav Pal, Sumantra R. Kundu, Amin R. Mazloom, and Sajal K. Das \\ Dept. of Computer Science and Eng. \\ University of Texas at Arlington \\ Arlington, TX 76019 - USA \\ $\{$ kundu, spal, mazloom, das\}@cse.uta.edu
}

\begin{abstract}
Current scheduling techniques used for cellular networks do not suffice for the emerging multi-rate systems like cdma2000 and High Data Rate (HDR). Real-time applications like video streaming must comprehend the channel conditions and consequently the data rates that are currently being supported; accordingly the content and the amount of data to be transmitted needs to be adapted to the available bandwidth. In this paper, we have considered multimedia (MPEG-4) streaming as the application over HDR and propose a content aware scheduling scheme (CAS) that takes into consideration the different priorities of the MPEG-4 stream content. The proposed transmission scheme considers both the channel conditions as perceived by the user as well as the priority of the streams. In addition, CAS verifies the playout timestamp and discards stale packets ensuring higher throughput in the process. We capture the lag of the proposed adaptation scheme using the KullbackLeibler distance and show that the rate adaption scheme has a reasonably small lag. Simulation results demonstrate that the proposed scheme results in higher overall peak signal to noise ratio (PSNR) values of the entire movie, lesser number of dropped frames, and a better throughput utilization over existing schemes.
\end{abstract}

\section{Introduction}

The advent of multi-rate systems like cdma2000 [1], HDR [2], and EDGE [3] provide higher bandwidth but pose challenges in the channel rate estimation and scheduling. Although advanced video standards like MPEG-4 and H.264 can take advantage of the increased transmission bandwidth and offer powerful error resilient mechanisms, they are unable to handle the complexities like attenuation due to multi-path fading, shadowing, transmission errors, bandwidth fluctuations, spectrum scarcity associated with the wireless channel. In this paper, we focus on the scheduling aspect for such systems.

In addition to round robin (RR) and first come first serve (FCFS) scheduling policies, video scheduling over HDR [6] have used Modified-Largest-WeightedDelay-First (M-LWDF) and Exponential (EXP) scheduling algorithm and variations of the two to reduce the percentage of video frames that do not meet

\footnotetext{
^ This work is supported by NSF ITR grant IIS-0326505.
} 
the playback deadlines. However, even such sophisticated radio resource management schemes are unaware of the content, priority and timing requirements demanded by the video streams.

In this paper, we provide rate adaptation techniques at the base station that adapts the transmission of the packets to the available bandwidth. It also ensures that packet buffer overflow/underflow does not take place at the client end. We also propose a scheduling algorithm specifically for streaming multimedia (MPEG-4) that not only adapts to the available bandwidth but also schedules the packet with respect to their priority for ensuring smooth video playout at the client end. On favorable channel conditions, the scheme transmits more packets than necessary for current viewing but honors the buffer overflow condition determined by the rate adaptation algorithm. The success of the proposed scheme is due to the priority structure of the MPEG-4 stream in which I frames have higher priority than $\mathrm{B}$ and $\mathrm{P}$ frames, since without the $\mathrm{I}$ frames video playout is not possible. Based on the channel conditions, the scheme not only adjusts the data but also drops the lower priority frames (in case of video), if necessary. We employ the Kullback Leibler 4 ] distance as a metric to measure the adaptation rate.

In summary, the main contributions of this paper are as follows:

- A rate adaptation scheme which takes into consideration the buffer overflow and underflow problems at the client end.

- A content aware scheduling technique which ensures that higher priority MPEG-4 frames are transmitted prior to the lower priority ones and takes into consideration the playout timing requirements.

- Experimental evaluation of the scheme using a framework consisting of Darwin Streaming Server (DSS), click modular router, and Mplayer as the client end media player reveals that the proposed technique achieves lower frame drop and higher PSNR values compared to the case with no content aware scheduling mechanism.

The rest of the paper is organized as follows: We discuss the necessary background on HDR systems and MPEG-4 in Section 2. A rate adaptation scheme based on the estimated channel throughput is proposed in Section 3 . Section 4 presents a case study of transmission of MPEG-4 over HDR using the proposed techniques. In Section [5] simulation results involving rate adaptation, scheduling algorithms, and performance of MPEG-4 transmission over HDR system are presented. Conclusions are drawn in the last section.

\section{Background}

A brief description of the HDR system model and the functioning of MPEG-4 is presented in this section.

\subsection{HDR System Model}

We consider a single cell of a multi-rate wireless system with the base station serving $N$ mobile terminals. We assume that the system employs data rate 
control mechanism on the forward link that adapts to the changing channel conditions by employing adaptive modulation and coding techniques, hybrid Auto Repeat-reQuest (ARQ), and best serving sector selection. The mobile terminals perform measurement of the current channel conditions (i.e., $\frac{E_{b}}{I_{o}}$ the received energy per bit to interference) and predicts the achievable rate. Every mobile terminal updates the base station of the predicted rate via the pilot signal on the reverse link data rate control (DRC) channel. At any time slot $t$, the data rate that can be supported by the $i$ th mobile terminal is $R_{i}(t), 1 \leq i \leq N$, where $R_{i}(t)$ is one of the many rates supported by the system. For example, HDR supports 11 data rates $\left[2 . \overline{R_{i}}(t)\right.$ is the mean rate actually provided to user $i$ measured over a sliding window of length $t_{c}$ and is given by

$$
\overline{R_{i}}(t+1)=\left(1-\frac{1}{t_{c}}\right) \times \overline{R_{i}}(t)+\frac{1}{t_{c}} \times R_{i}\left(t_{c}\right)
$$

This cumulative estimation is put into effect at each slot; however the scheduling step is executed once every new transmission.

\section{Rate Adaptation in Multi-rate Systems}

We initially present the adaptation algorithm and thereafter present the analytical modeling of the adaptation scheme and propose adaptation metrics using the Kullback Leibler distance. The process of adaptation of the application bit stream in response to the available transmission rate gives rise to the following three scenarios. We denote $r_{i}(t), X_{b}(t)$, and $X_{e}(t)$ as the available transmission rate, the required base layer bit rate, and the enhanced layer bit rate (applicable only for FGS MPEG-4) respectively.

- Case $I: r_{i}(t)<X_{b}(t)$ : The adaptation scheme selectively drops base layer frames based on the proposed prioritization scheme.

- Case II: $X_{b}(t) \leq r_{i}(t) \leq X_{b}(t)+X_{e}(t)$ : The highest priority layer is encoded. For the enhanced layer, the adaptation scheme encapsulates whatever portion of the EL that can be packed into the remaining available bandwidth.

- Case III: $r_{i}(t)>X_{b}(t)+X_{e}(t)$ : This is the most favorable case where the encoding rate is higher than the bit stream rate and hence the entire data can be transmitted.

Through the proposed adaptation scheme, the lowest possible granularity is achieved. However, a finite lag remains between the channel state and actual transmission of the data which in our case is the best achievable, i.e., lag by a slot. Note that during packetization the granularity is measured in terms of the decision period. Depending on the current buffer status at both the transmitter and user and the current channel state, the rate control system needs to determine the encoding rate. The currently encoded packets would only be served after the existing MAC PDU's are served. Thus, the rate control system needs to determine the number of decision periods required to serve the existing MAC PDUs. Hence, prediction of the rates by transition probability computation is important which we discuss next. 


\subsection{Transition Probability Calculation}

We assume that the data rates are related to the distance of the user from the base station [13. Though this assumption holds true under ideal conditions (i.e., no interference, no topological effects), we still use it for mathematical simplicity. If we assume that the system supports $m$ rates, then a cell can be divided into $m$ concentric rings; the innermost ring gets the maximum data rate and outermost ring gets the minimum. In one decision period, a user can go one ring above or below the current ring because of the limitation in speed. Let $P_{u v}$ denote the probability of a user switching from a data rate $u$ to data rate $v$. Note, $v$ can only be $u-1, u$, or $u+1$. Let the steady state probability of an user being in the $u$ th data rate be denoted by $\pi_{u}$. We define $\pi_{u}$ as the ratio of the ring area by the cell area, and is given by $\pi_{u}=\frac{r_{u}^{2}-r_{u-1}^{2}}{r_{m-1}^{2}}$ where $r_{u}$ is the radius of the $u$ th ring. Clearly, $\sum_{u=0}^{m-1} \pi_{u}=1$. The transitional probabilities $P_{u v}$ are computed geometrically and can be given as follows

$$
\begin{aligned}
P_{u \rightarrow u-1} & =\frac{2 \sqrt{s\left(s-r_{1}\right)\left(s-r_{2}\right)(s-d)}-\left(\theta_{1} r_{1}^{2}-\theta_{2} r_{2}^{2}\right)}{\pi r_{2}^{2}} \\
P_{u \rightarrow u} & =\frac{A_{i}}{\pi r_{2}^{2}} \\
P_{u \rightarrow u+1} & =\frac{A_{b g e}}{\pi r_{2}^{2}}
\end{aligned}
$$

Note that these probability calculation will be different for the inner most and outer most rings.

\subsection{Analytical Modeling of Rate and Adaptation}

The encoding rate $r_{i}(t)$ for user $i$ depends on the channel state, buffer status, and bandwidth allocated. This necessitates the computation of the number of decision periods needed to serve the existing MAC PDUs. The number of decision periods is basically the lag by which the encoder follows the channel state. If $\xi$ be the number of decision periods needed to serve the MAC buffer $B_{i}$, then

$$
\sum_{l=0}^{\xi} S_{i}^{l} \times P_{u v}^{l} \times R_{i}^{l}(t)=f_{b} \times B_{i}
$$

where $0 \leq f_{b} \leq 1$ is the buffer fullness, $S_{i}^{l}$ is the number of slots allocated to user $i, P_{u v}^{l}$ is the transition probability from state $u$ to $v$, the calculation of which can be found in [2. $R_{i}^{l}(t)$ is the rate estimated using Equation 1] all in the $l$ th decision cycle.

We need to compute the amount of data $\left(\chi_{i}(t)\right)$ that the MAC scheduler would be able to support for the corresponding decision period. Depending on the scheduling paradigm which determines the number of slots being allocated to the user, the amount of data which the adaptation layer may encode is given by

$$
\chi_{i}(t)=S_{i}^{\xi} \times P_{u v}^{\xi} \times R_{i}^{\xi}(t)
$$


Conversely, if the mobile terminal undergoes favorable channel condition i.e., would support a higher data rate than the rate controller would be tempted to transmit more data provided the receiver buffer does not overflow. Transmitting more data than required would compensate if channel conditions deteriorate. The available buffer space $\left(v_{i}(t)\right)$ during that decision period is

$$
\begin{aligned}
v_{i}(t)=\left(1-f_{r b}\right) \times R B_{i}- \\
\quad \sum_{l=0}^{\xi}\left(S_{i}^{l} \times P_{u v}^{l} \times R_{i}^{l}(t)\right)+\rho_{i} \times(\tau \xi)
\end{aligned}
$$

where $0<f_{r b}<1$ is the receiver buffer fullness, $R B_{i}$ is the receiver buffer size, $\rho_{i}$ is the rate of the playout curve rate, and $\tau$ is the time of each slot $(\tau=1.67 \mathrm{~ms}$ for HDR).

The adaptation layer encoding rate, considering the buffer constraints at both the transmitter and receiver sides is

$$
r_{i}(t)=\min \left(\chi_{i}(t), v_{i}(t)\right)
$$

Note that $r_{i}(t)$ is a random variable which gives the value of rate adaptation. Let $p(\cdot)$ denote the pdf for the random variable $r_{i}(t)$. Let $f_{g}(\cdot)$ denote the pdf of the Group of Picture (GoP) size distribution of the $g$ th video. We use the Kullback Leibler (KL) distance to characterize the performance of our adaptation scheme.

Definition: Kullback Leibler distance which determines the relative entropy between two distributions is given as

$$
D(p \| q)=\sum_{x \in X} p(x) \ln \frac{p(x)}{q(x)}
$$

$D(p \| q)$ is the measure of the inefficiency by which the distribution $q(x)$ differs from distribution $p(x)$. Hence Equation (7) provides a metric to determine the lag or closeness of $q(x)$ to $p(x)$. Substituting $p(x)=p(\cdot)$ and $q(x)=f_{m}(\cdot)$, we define that the adaptation $(\mathcal{A})$ of the video to channel rate as

$$
\mathcal{A}\left(p \| f_{m}\right)=\sum p(.) \ln \frac{p(.)}{f_{m}(.)}
$$

The granularity of the rate of adaptation is bounded by the decision period as given in Equations (3), (4), (5) and (6). Hence we analyze the adaptation with respect to the decision period. The time granularity of the decision period is denoted by $s$.

\section{MPEG over Multi-rate System}

The success of video streaming depends on devising mechanism to adapt to the changing channel conditions. The popular techniques for TCP rate adaptation [7] 
did not consider the fluctuation in channel conditions. Numerous link layer schemes 89. exist for enhancing TCP over wireless networks. But link layer aware techniques [1011] use statistical measure of packet loss for adaptation. These techniques fail to capture the content level information which needs to be exploited for video applications. It is known that better rate control algorithms are obtained if stochastic channel behavior through a priori models are considered [12. We incorporate the channel model as well as the video information in our rate adaptation algorithm at the MAC layer. The scheduler transmits more data than necessary when the conditions are favorable, whilst avoiding buffer overflow. Whereas it drops frames without compromising on the integrity of the video when the conditions are degraded. For example, successful transmission of I-frames is more crucial than others. This knowledge of frame priority at the MAC layer helps the rate adaptation. This is because the delay in retransmission of I-frames from the application layer is higher than retransmitting from the MAC layer. In addition, the MAC layer can drop less important frames based on the priority and closely follow the channel conditions by minimizing the Kullback-Leibler distance.

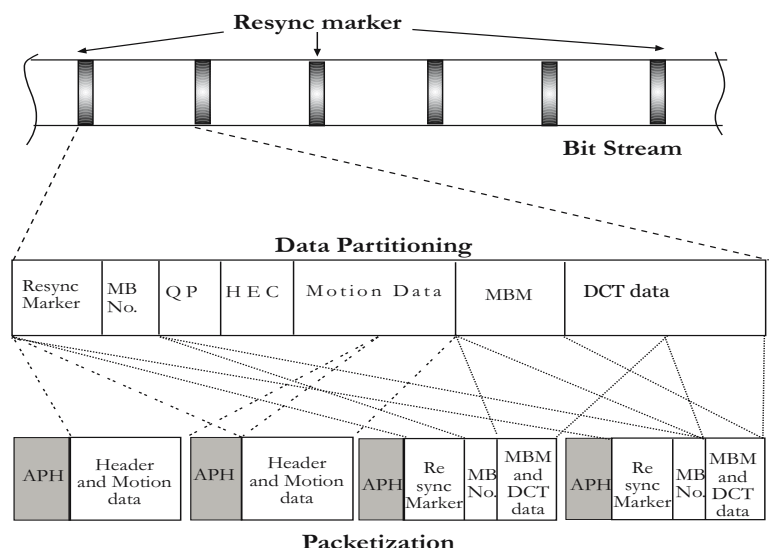

Fig. 1. Hierarchical Packetization of Video Packets

\subsection{Fragmentation, Packetization, Prioritization Layer (FPPL)}

In general applications the working of these layers are mostly static in nature and do not consider the underlying network protocols or the possible variation in the channel conditions. We propose a hierarchical fragmentation, packetization and prioritization layer (FPPL), specifically designed for multi-rate wireless systems, that uses the packet resynchronization and exploits the underlying MAC and the channel conditions for packetization/fragmentation.This hierarchy is shown in Figure 1 . 
Since FPPL is MAC-aware, the FPPL packet size is matched to the MAC protocol data unit (PDU) to prevent further fragmentation at the MAC layer.

\subsection{Radio Resource Scheduling}

The MAC Scheduler (MAC-S) is responsible for allocating slots among the users requiring different data rates. It can be any generic scheduler like the proportional fair scheduler. These standard MAC schedulers do allocate slots either based on the supportable channel rate or/and by the QoS demanded by each type of user but unfortunately is unaware of the content type being served from the MAC buffer. Hence, the MAC-S is incapable of exploiting the application specific features while scheduling. To overcome the shortcomings of the MAC-S scheduler, we propose a Content Aware Scheduler (CAS) as shown in Figure 2 which works in conjunction with the MAC-S.

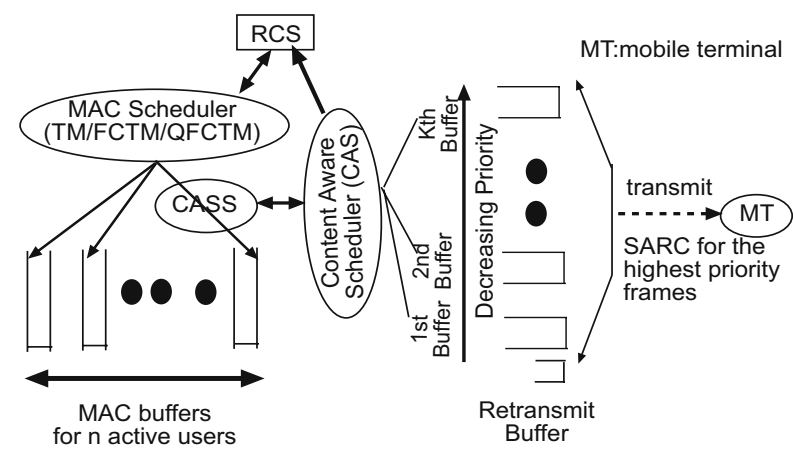

Fig. 2. MAC-S and CAS Schedulers

Depending on the application the FPPL generates the appropriate number of priority levels and performs fragmentation and packetization accordingly. We assume that $k$ can be any number of priority levels depending on the application. The CAS models the MAC buffer into $k$ number of queues one for each priority level. For example in case of the FGS MPEG-4 video data there would be 4 queues one each for the I, B, P-frames and the last one for the enhancement layer with the highest priority being given to the I-frames and the least to the enhancement layer. The generic MAC-S does not differentiate the priorities and simply serves the content in a first come first serve (FCFS) queuing discipline. However, the proposed CAS being content aware enhances the system goodput. In many cases for real time streaming applications, transmission of the higher priority content is essential for the success of the application. In addition, the proposed CAS is aware that the packets need to be transmitted before a certain deadline at the receiver side. The CAS is smart enough to discard packets if stale. 
For $i$ th packet, we define a boolean variable $S P(i)$ which determines whether the packet is stale or not. A packet $i$ is defined stale if $S P(i)=1$ and the condition is $t_{\text {curr }}>T S_{i}+t_{\text {prop }}$ else $S P(i)=0$ meaning the packet is not stale; where $t_{\text {curr }}$, $T S_{i}$ and $t_{\text {prop }}$ are the current system time, the time stamp of packet $i$ prior to which it needs to be transmitted and the propagation time, respectively. This simple yet content aware scheduling mechanism prevents error propagation and increases the goodput.

The MAC-S is unaware of the prioritization scheme and is unable to schedule efficiently. However, the CAS utilizes the priority information and also employs a Selective Adaptive Retransmit Control (SARC) mechanism for the highest priority packets. The SARC mechanism can be utilized till any level of priority i.e. for each buffer but for the sake of simplicity we restrict ourselves to the highest priority buffer. Additionally, the CAS employs Fast Transmit Scheme (FTS) to take advantage of favorable channel condition but does take into account of the upper limit Thus, in summary, the rationale for employing SARC are the following:

1. Provide unequal error protection (UEP) to high priority data and thereby increase the transmission quality and prevent error propagation.

2. Mobile terminal's buffer fullness $\left(f_{r b}\right)$ is transmitted back to the base station through the SARC mechanism. $f_{r b}$ is essential for boundary condition determination.

The SARC mechanism of CAS keeps retransmitting the highest priority till the mobile terminal acknowledges the successful arrival of the frame. The number of retransmissions is bounded by the timing requirements, i.e., till the packet becomes stale. Continuing with the FGS MPEG-4 example the I-frame being the most important video data frame, multiple retransmissions might be required for it. Missing or corrupted I-frame results in wastage of the corresponding B, $\mathrm{P}$ and EL frames even if they arrive correctly. The FTS scheme enables the CAS to transmit more data than the playout curve rate of the mobile terminal when the channel conditions are favorable provided CAS has assigned sufficient slots by the MAC-S. Since the receiver buffer of the mobile terminal is finite, the CAS should restrain from transmitting data that would overflow the mobile terminal buffer. Similar to the ARQ mechanism in TCP, the mobile terminal transmits the available buffer space $(\beta)$ in each acknowledgment packet. The CAS makes the rate control system module aware of $\beta$. Thereafter, RCS module takes into account of the available rate, buffer space at both the $\mathrm{MAC}$ and mobile terminal, and computes the upper bound for data transmission. It also determines whether the fast transmission scheme outlined earlier is achievable or not. The working principle of CAS is explained below. Without loss of generality let us explain the CAS scheduling of an application having $k$ priority levels whose queues denoted by $i H P$ where $i \epsilon\{1,2, . ., k\}$. The highest priority level enabled with SARC in the $K^{t h}$ decision cycle of the MAC-S scheduler. 


\section{Scheduling Algorithm $C A S()$}

1: CAS $\leftarrow$ control state

2: if (Overflow Constraint not violated) then

3: $\quad$ if $1 \mathrm{HP}$ not empty then

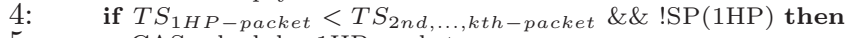

5: $\quad$ CAS schedules 1HP-packets

6: CAS employs SARC

//wait for $\zeta$ slots for ACK

Return

end if

end if

if $\mathrm{CAS} \leftarrow \mathrm{SARC}$ feedback then

Retrieve retransmit packet numbers

Compute Overflow Constraint

if ! $\mathrm{SP}(1 \mathrm{HP}$-packet) then

CAS retransmits $1 \mathrm{HP}$-packets

CAS employs SARC

if slot available then

pack $2_{n d} \mathrm{HP}, \ldots, k_{t h} \mathrm{HP}$-packets respectively

//wait for $\zeta$ slots for ACK

end if

Return

end if

end if

$\mathrm{i}=2$

if $i_{t h}$-Buffer not empty then

CAS transmits $i_{t h}$ Buffer packets.

Increment $\mathrm{i}$

end if

27: end if

CAS initiates transmission with the highest priority packets employing Selective Adaptive Retransmit Control and following up with the rest of the frames without employing any ARQ on the rest of the packets. For CAS, goodput would be more effective measure than throughput. We define goodput for real time data as the number of packets transmitted per decision cycle by the CAS scheduler that the mobile terminal successfully utilizes. The calculation of CAS goodput is done in section 4.3 .

\subsection{CAS Goodput}

In this section, we perform the analytical modeling of the Content Aware Smart Scheduler and also derive the goodput of the system using CAS. First, we model the SARC mechanism. Let $P_{b}(m)$ and $P_{e}(m)$ denote the probability of the bit error and packet error respectively for sending $m$ packets simultaneously. If $P_{s}(m)$ denotes the probability of successful packet transmission then

$$
P_{s}(m)=1-P_{e}(m)=\left[1-P_{b}(m)\right]^{L}
$$

where $L$ denotes the length of the MAC protocol data unit (PDU). To provide more protection to the highest priority packets, error correction coding (ECC) is employed. The modified probability for packet transmission with ECC at any point of time is

$$
P_{s}(m, y)=\sum_{i=0}^{y}\left(\begin{array}{l}
L \\
o
\end{array}\right) P_{b}(m)^{i}\left[1-P_{b}(m)\right]^{L-o}
$$


where $o$ is the number of errors corrected and $y$ is the total number of correctable bit errors. Thus $P_{s}(m, y)$ denotes the probability for successful packet transmission for highest priority packets. However, the number of retransmissions for these packets is limited by $\epsilon$, the maximum number of retransmits for the $i^{\text {th }}$ $1 \mathrm{HP}$ packets. We compute $\epsilon$ as

$$
\epsilon=\frac{T S_{1 H P-\text { packet }}}{t_{\text {prop }}+t_{\text {arq }}} \text { if } \operatorname{SP}(1 \mathrm{HP} \text {-packet })
$$

where $t_{\text {prop }}, t_{\text {arq }}$ and $T S$ denote the propagation, the time after which the ACK transmitted by the mobile terminal is received, and the timestamp of the corresponding packet before being marked stale, respectively. The mean number of retransmissions for $1 \mathrm{HP}$-packets is given by

$$
\begin{aligned}
\delta_{1 H P} & =\sum_{i=1}^{\epsilon}\left(1-P_{s}\right)^{(i-1)} P_{s} \times i \\
& =P_{s} \times \frac{1-P_{s}^{\epsilon}}{\left(1-P_{s}\right)^{2}}
\end{aligned}
$$

Let $n_{k}$ be the total number of MAC-PDUs generated by FPPL for the $k^{t h}$ data segment and let $n_{k 1 H P}$ and $n_{k R}$ be the number of 1 HP-frame packets and $2, \ldots, k$ - frame packets respectively such that $n_{k}=n_{k 1 H P}+n_{k R}$. The timestamp of the frames is dependent on the data size distribution. We model $\delta_{R j}$ as the parameter which determines whether $2, \ldots, k$ - frame are stale. Consider that $\delta_{R j}=\mathrm{SP}(\mathrm{j})$, thus the effective number of packets transmitted per decision period by CAS is given by

$$
\Delta_{i}=n_{k I} \times \delta_{I}+n_{k R} \times \delta_{R j}
$$

Therefore, the average goodput $\left(\rho_{g p}\right)$ of CAS is given by

$$
\rho_{g p}=\frac{\sum_{i=1}^{K} \Delta_{i}}{K}
$$

where $K$ is the total number of decision periods for which video is transmitted. Note that throughput of such systems would fail to capture the actual system performance since it would not consider adaptive selective retransmission and selective frame dropping based on packet staleness.

\section{Simulation Results}

We have conducted simulation experiments where a single multi-rate cell with multiple users was simulated to illustrate the performance of the proposed adaptation technique with respect to the existing scheme for HDR. The video test sequences chosen comprises of both the simple profile (SP) and advanced simple profile $(\mathrm{ASP})$. In order to ensure the performance of the proposed schemes, we use representative test sequences of foreman, paris and football which have 
Table 1. Specification of the files used in our simulation

\begin{tabular}{|c|c|c|c|}
\hline Resolution & Clip name & fps & bitrate \\
\hline QCIF & paris & $15 \mathrm{fps}$ & $64 \mathrm{kbps}$ \\
\hline QCIF & foreman & $25 \mathrm{fps}$ & $64 \mathrm{kbps}$ \\
\hline CIF & football & $15 \mathrm{fps}$ & $1 \mathrm{Mbps}$ \\
\hline
\end{tabular}

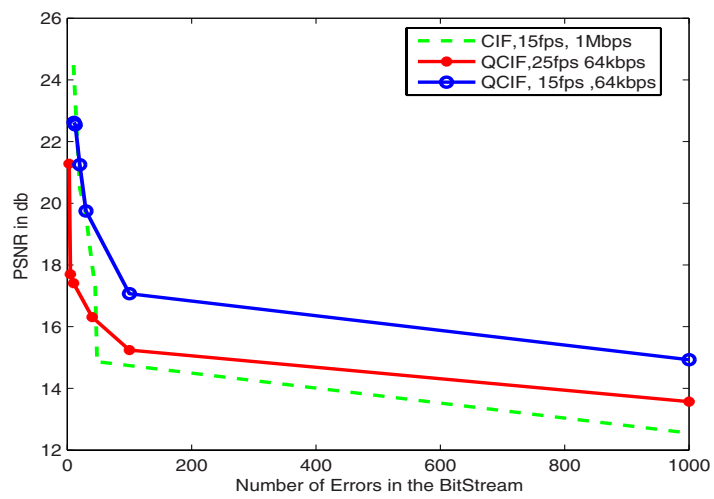

Fig. 3. Observe the smooth variation of PSNR with BER using our CAS algorithm

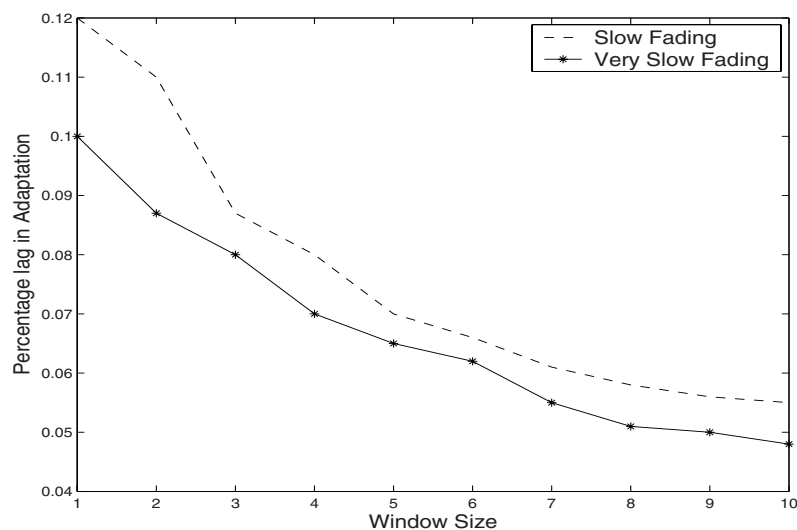

Fig. 4. The variation of CAS adaptation due to mobility

varying resolution, frame rate and bit rate. The specifications of the streams are listed in Table 1 .

Figure 3 highlights the graceful degradation of PSNR values using the CAS scheduler. As for the lag in adaptation, we analyzed equation 8 numerically. Figure 4 shows how the system is able to learn and adapt if a sufficiently long window of observation is allowed. As we do not deal with fast fading channels, we compared 'slow' and 'very slow' fading channels. Obviously, the adaptation is 


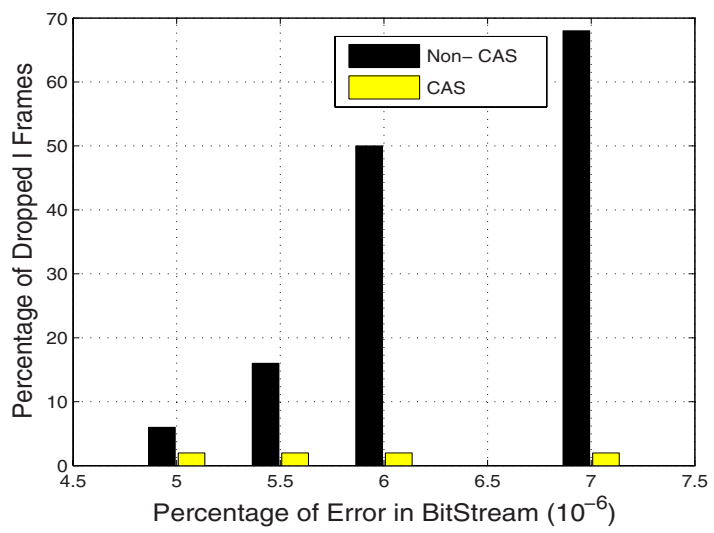

Fig. 5. Improved throughput of I-frames due to CAS. The data transmission quality by CAS is also impressive and lesser bits are flipped due to adaptability nature of the CAS.

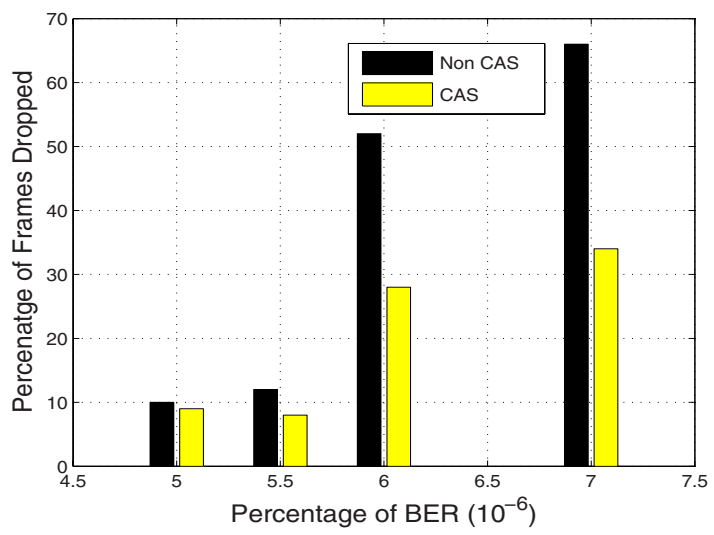

Fig. 6. Error resiliency of CAS and throughput of I-frames. As the channel condtion degrades the CAS shows significantly better adaptability to erroneous medium and results fewer Ipacket drops.

better for slower fading channels. In Figures 5 and 6 , we show how the throughput of I-frames in multimedia streaming could be improved in the presence of CAS.

\section{Conclusion}

This paper deals with rate adaptation at the MAC layer which is necessary for streaming multimedia over multi-rate wireless systems. We propose a rate adaptation technique where the application layer encoding rate is dynamically adjusted to the varying channel conditions perceived by the user. We have also enhanced the fragmentation, packetization and prioritization scheme for FGS MPEG-4 and tailored it for the multirate wireless systems. We have shown how content-aware 
scheduling at the MAC layer can enhance the performance for video data transmission by selectively deciding on the nature and importance of the content.

\section{References}

1. 3GPP2 C.S0024 Ver3.0, "cdma2000 High Rate Packet Data Air Interface Specification", Dec. 5, 2001.

2. P. Bender, P. Black, M. Grob, R. Padovani, N. Sindhushayana, and A. Viterbi, "CDMA/HDR: A Bandwidth-Efficient High-Speed Wireless Data Service for Nomadic Users", IEEE Comm. Magazine, July 2000.

3. A. Furuskar, S. Mazur, F. Muller and H. Olofsson, "EDGE: Enhanced Data Rates for GSM and TDMA/136 Evolution," IEEE Personal Communication Magazine, pp 56-66, June 1999.

4. S. Kullback and R. A. Leibler, "On information and sufficiency", Ann. Math. Stat., 22:7986, 1951.

5. R. Koenen, "Overview of the MPEG-4 Standard", ISO/IEC JTCI.SC29/WG11 M4030, 2001.

6. K. Gribanova and R. Jntti, "On Scheduling Video Streaming Data in the HDR System", Vehicular Technology Conference, 2004.

7. M. Chen and A. Zakhor, "Rate Control for Streaming Video over Wireless", Infocom, 2004.

8. H. Balakrishnan and R. Katz, "Explicit Loss Notification and Wireless Web Performance", IEEE Globecom Internet Mini-Conference, 1998.

9. S. Biaz, N. H. Vaidya, "Distinguishing congestion losses from wireless transmission losses: a negative result", Computer Communications and Networks, 1998.

10. H. Balakrishnan, V. N. Padmanabhan, S. Seshan and R. H. Katz, "A Comparison of Mechanisms for Improving TCP Performance over Wireless Links", Proc. ACM Sigcomm 1996, Stanford, CA, August 1996.

11. S. Cen, P. C. Cosman, and G.M. Voelker, "End-to-end differentiation of congestion and wireless losses", Proc. Multimedia Computing and Networking (MMCN) conf. 2002, pp. 1-15, San Jose, CA, Jan 23-25, 2002.

12. S. Aramvith, I. Pao and M. Sun, "A Rate-Control Scheme for Video Transport over Wireless Channels", IEEE Transactions on Circuits and Systems for Video Technology, Vol. 11, no. 5, May 2001.

13. T. Bonald and Alexandre Proutiere, "Wireless downlink data channels: user performance and cell dimensioning", Proceedings of the 9th annual international conference on Mobile computing and networking, San Diego, CA, USA.

14. A. Jalali, R. Padovani, and R. Pankaj, "Data throughput of CDMA-HDR a high efficiency-high data rate personal communication wireless system," in IEEE Proc. of Vehicular Technology Conf. 2000-Spring. 2000, vol. 3.

15. L. D. Soares and F. Pereira, "MPEG-4: a flexible coding standard for the emerging mobile multimedia applications", IEEE Int. Symp. on Personal, Indoor and Mobile Radio Communications, Volume: 3, pp. 1335-1339, 1998.

16. W. Chung, H. W. Lee, and J. Moon, "Downlink Capacity of CDMA/HDR"', in the Proc. IEEE 2001 Vehicular Technology Conference (VTC2001-Fall), Atlantic City, NJ, USA, October 2001.

17. C. Bormann, L. Cline, G. Deisher, T. Gardos, C. Maciocco, D. Newell, J. Ott, G. Sullivan, S. Wenger, and C. Zhu, "RTP Payload Format for the 1998 Version of ITU-T Rec. H.263 Video (H.263+)," Internet Engineering Task Force, RFC 2429, Oct. 1998.

18. T. V. Laksman, A. Ortega and A. R. Reibman, "VBR video: Trade-offs and potentials," Proc. IEEE, vol 86. pp. 952-973, May 1998. 